\title{
Novel polybasic cleavage site in SARS-CoV-2 genome is likely to induce a major change in the RNA secondary structure
}

Amirhossein Manzourolajdad ${ }^{1}$, Zhenming $\mathrm{Xu}^{2}$, Diako Ebrahimi ${ }^{3^{*}}$

${ }^{1}$ RNA Molecular Biology Group, Laboratory of Muscle Stem Cells and Gene Regulation, National Institute of Arthritis and Musculoskeletal and Skin Diseases, National Institutes of Health, 50 South Drive, Bg 50 Rm 1152, Bethesda, MD 20814, USA. amir.manzourolajdad@nih.gov

${ }^{2}$ Department of Microbiology, Immunology and Molecular Genetics, Long School of Medicine, University of Texas Health Science Center at San Antonio, 7703 Floyd Curl Drive, San Antonio, TX 78229, USA. XuZ3@uthscsa.edu

${ }^{3}$ Texas Biomedical Research Institute, 8715 W Military Dr, San Antonio, TX 78227, USA. debrahimi@txbiomed.org (*Corresponding author) 


\begin{abstract}

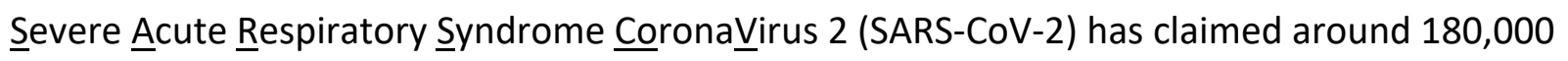
lives and continues to spread. There are currently no approved medications or vaccines for this new coronavirus. Studies have shown that the positive RNA genome of SARS-CoV-2 contains unique features, including a 12-base sequence inserted between the two subunits of viral receptor protein Spike. This inserted sequence facilitates the cleavage of Spike by the cellular proteases Furin and TMPRSS2, leading to the fusion of virus and host cell membranes. Current studies are mostly focused on the SARS-CoV-2 Spike protein and its interacting cellular proteins ACE2, Furin, and TMPRSS2. RNA structural studies are limited and little is known about the potential impact of the 12-base sequence insert on the secondary structure of SARS-CoV-2 genomic RNA and/or its transcripts. Here, by using local and global RNA secondary structure predictions, we show that the novel 12-base insert of SARS-CoV-2 genome likely induces a major RNA secondary structure change.
\end{abstract}




\section{Introduction}

The reported number of people infected with SARS-CoV-2 is approaching 2.6 million, over 177,000 of whom have lost their lives. However, recent seroprevalence data show that the infection rate is likely 50-85 folds higher ${ }^{1}$. Investigators from a broad spectrum of scientific disciplines are joining forces and sharing data and resources to better understand the complex biology of this new coronavirus and expedite the development of therapeutic and/or prevention strategies. Nevertheless. The SARSCoV-2 genome has at least two unique features, with likely roles in the high rate of transmission of this virus and its leap into humans ${ }^{2}$. Both of these two features reside within the viral Spike (S) gene, one encoding the receptor binding domain (RBD) with an enhanced affinity to the human cell surface receptor ACE2, and the other encoding an inserted polybasic cleavage site. The Spike proteins, which decorate the outer layer of SARS-CoV-2 virions have two subunits, the N-terminal S1 and the C-terminal S2. Viral entry is initiated by the interaction between the Spike RBD within the S1 subunit and ACE2 ${ }^{3-5}$. This interaction is followed by fusion of viral and cell membranes mediated by the fusion peptide located in the C-terminal S2 subunit, a step critical for infection of all coronaviruses ${ }^{6,7}$. The RBD of SARSCoV-2 Spike is unique in that it resembles the binding domain of the SARS-like coronaviruses found in pangolin (98\% amino acid identity). By contrast, most other regions of the SARS-CoV-2 genome are closely similar to a bat coronavirus known as Bat-RaTG13 ${ }^{8}$. The RBD of Bat-RaTG13 is only $81 \%$ identical to that of SARS-CoV-2. Therefore, pangolin is currently considered the intermediate species for the transmission of the new coronavirus from bats to humans ${ }^{9}$.

The second unique feature of SARS-CoV-2 genome is an inserted 12-base sequence (CCUCGGCGGGCA) between the S1 and S2-coding sequences ${ }^{9,10}$. This insertion, which codes for four amino acids PRRA, has not been observed in any other similar coronaviruses. The last three amino acids (RRA) are part of a sequence known as the polybasic cleavage site (RRAR) ${ }^{2}$, which is a substrate for protease cleavage of Spike into the S1 and S2 subunits ${ }^{11-13}$. The cellular protease Furin has been shown to be responsible for this cleavage, which is necessary for cell-cell fusion and virus-cell fusion mediated by Spike ${ }^{14}$. However, the precise cellular location and mechanism of S1/S2 cleavage are not fully understood. A separate study of SARS-CoV-2 cultures in Vero-E6 cells has identified variants with S1/S2 junction deletions spanning the 12-base motif CCUCGGCGGGCA ${ }^{15}$. Importantly, the deleted variants showed attenuated pathogenicity. Given that this 12-base sequence motif is intact in clinical isolates, it has been suggested that this region may be under selective pressure in infected patients ${ }^{15}$. Altogether, there is a growing body of evidence supporting a significant role for these inserted 12 bases in SARSCoV-2 biology. 
Studies so far, have focused, mostly, on understanding the role of these two SARS-CoV-2 features at a protein level. Recent RNA studies have reported several conserved and stable secondary structures ${ }^{16,17}$, however, little is known about the potential RNA secondary structure changes resulted from these novel sequence variations within the SARS-CoV-2 genome. Particularly, it is not clear if the RNA secondary structure is affected by the insertion of motif CCUCGGGGGCA. We note that this motif is highly GC rich and includes two CpG sites at positions 4 and 7. GC-rich regions have been reported to stabilize RNA secondary structures ${ }^{18-20}$. We, therefore hypothesize that the insertion of this 12-base motif leads to the formation of novel secondary structures in SARS-CoV-2 RNA. To test this hypothesis, we performed local and global RNA secondary structure predictions using a Minimum Free Energy (MFE)-based approach. Our analyses predict that the novel 12-base motif CCUCGGCGGGCA likely induces a major secondary structure change in SARS-CoV-2 RNA.

\section{Methods}

The SARS-CoV-2 reference genome (NC_045512, 29,903 bp, Severe acute respiratory syndrome coronavirus 2 isolate Wuhan-Hu-1) was used to make seven mutants (Table 1). These mutant sequences include different variants with alterations in the 12-base motif CCUCGGCGGGCA [nucleotide positions $23603-23614]$. Mutant $\Delta \mathrm{M}$ lacks the 12 -base motif, and other mutants include variations to alter the $\mathrm{CpG}$ sites (M1 and M2) or all 12-bases (MA, MC, MG, and MU). We used Vienna RNA software package ${ }^{21}$ for MFE-based RNA secondary structure prediction ${ }^{22}$. Full-length genomes were used to find the globally optimal RNA secondary structures. Structure visualization was done using the VARNA software package ${ }^{23}$.

Table 1. The nucleotide sequences of the 12-base motif in wild-type SARS-CoV-2 and seven mutants.

\begin{tabular}{|c|c|c|}
\hline Acronym & Length & Novel 12-base sequence motif \\
\hline WT & 29903 & CCUCGGCGGGCA \\
\hline$\Delta M$ & 29891 & $\Delta$ \\
\hline$M 1$ & 29903 & CCUCACGUAGCA \\
\hline M2 & 29903 & CCUCUGCAUGCA \\
\hline$M A$ & 29903 & AAAAAAAAAAAA \\
\hline MC & 29903 & CCCCCCCCCCCC \\
\hline MG & 29903 & GGGGGGGGGGGG \\
\hline MU & 29903 & UUUUUUUUUUUU \\
\hline
\end{tabular}




\section{Results}

The estimated global RNA conformations of wild-type and mutant SARS-CoV-2 sequences are shown in Figure 1. The red line shows the approximate location of the 12-base motif CCUCGGCGGGCA located inside the $S$ gene ${ }^{2}$. Two global RNA secondary structures (I and II) were predicted for these sequences. The wild-type RNA, $M 1$, and $M 2$ are predicted to have structure 1 , and mutants $\triangle M, M A, M C$, MG, and MU are predicted to have structure II (Figure 1). Figures 2A-D depicts the globally predicted secondary structures of WT and $\triangle M$ RNA around the 12-base motif, which is shown in red. The 12-base motif forms part of a stable stem structure (See SO in Figure 2C). As indicated, in the two globally stable structures of Figures $\mathbf{2 C}$ and $\mathbf{2 D}$, most of the base-pairings are intact between $\mathrm{WT}$ and $\Delta \mathrm{M}$. For example, stem-loops T1-T6 are present in both structures. However, the presence of the 12-base motif CCUCGGCGGGCA has led to the stabilization of additional structures, which are otherwise predicted not to exist (See bases colored in green in Figure $2 \mathrm{C}$ ). Figures $2 \mathrm{E}$ and $\mathbf{2 F}$ show the results of local RNA secondary structure predictions for WT and $\Delta \mathrm{M}$ in an extended region around the 12-base motif. As indicated, the structure estimated for SO (shown in red in Figure 2E) is identical in both locally optimum and globally optimum predictions (Figure 2C and Figure 2E). Similar to the global prediction, S0 does not appear in the locally optimal structure when the 12-base motif is removed (Figure 2F). Interestingly, certain predicted substructures in the vicinity of S0, such as $\mathrm{T} 1$, have been reported to be amongst conserved regions across SARS-CoV-2, SARS, and bat coronavirus sequences ${ }^{17}$. Altogether, our global and local model predicts that the removal of the 12-base motif CCUCGGCGGGCA from RNA or its replacement by a homopolymer sequence is associated with major secondary RNA structure changes. These changes include both short-range and long-range interactions as indicated in Figures $\mathbf{1}$ and $\mathbf{2}$. Examples of long-range interactions at the $5^{\prime}$ and $3^{\prime}$ of structure I are provided in Figure 1. By contrast, specific disruption of only CpG sites within the 12-base motif, as in mutants $M 1$ and $M 2$, is not predicted to have a significant effect on the RNA secondary structure. 

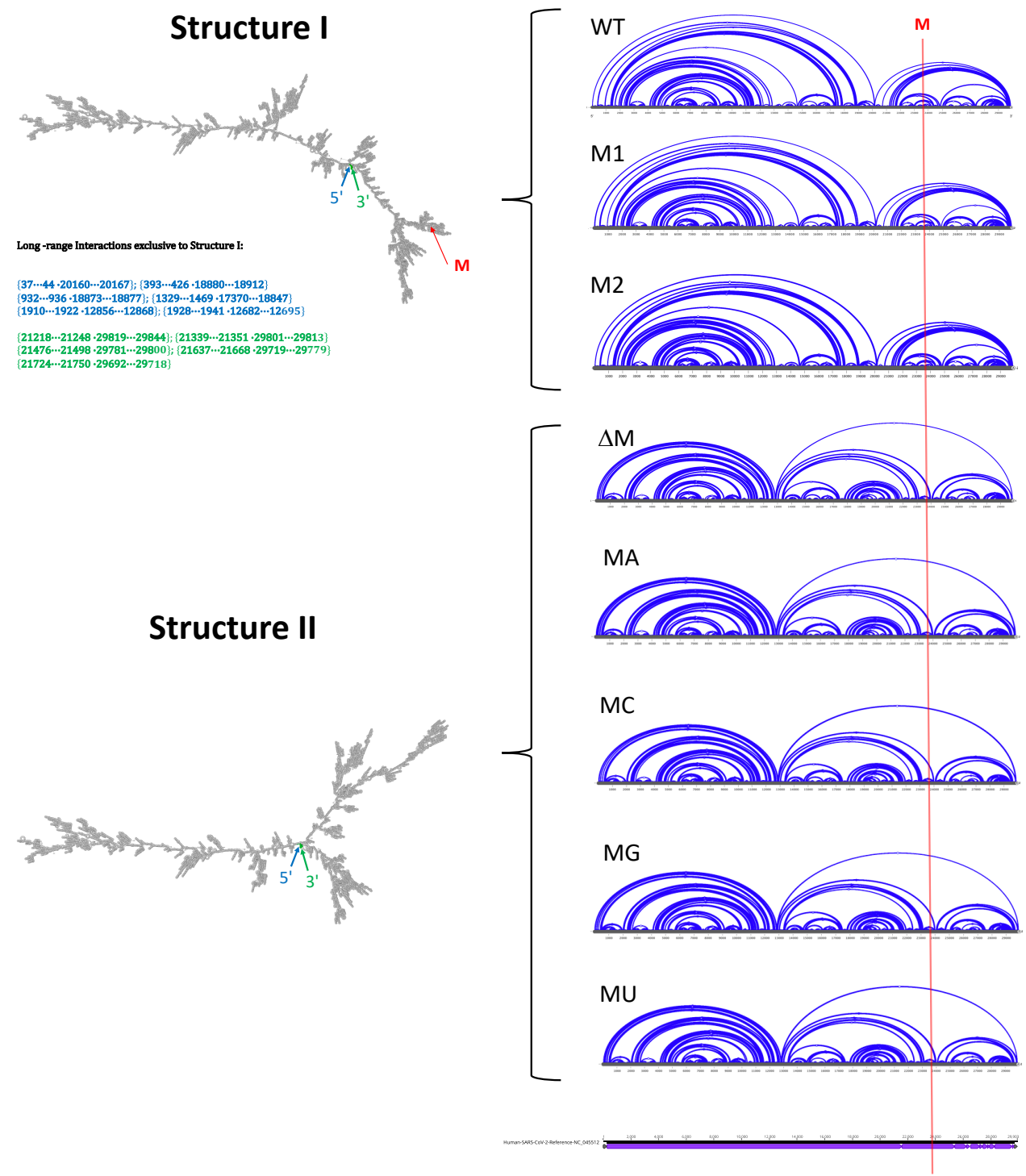

Figure 1. Predicted global secondary structure of wild-type and mutant SARS-CoV-2 RNA. The red line shows the proximal location of the 12-base motif CCUCGGCGGGCA [23603 - 23614]. 
A)

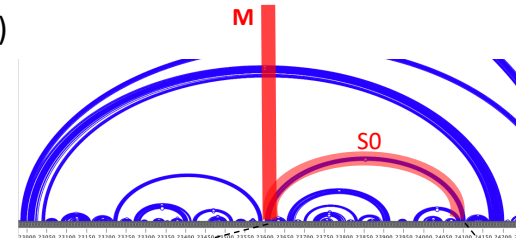

C)

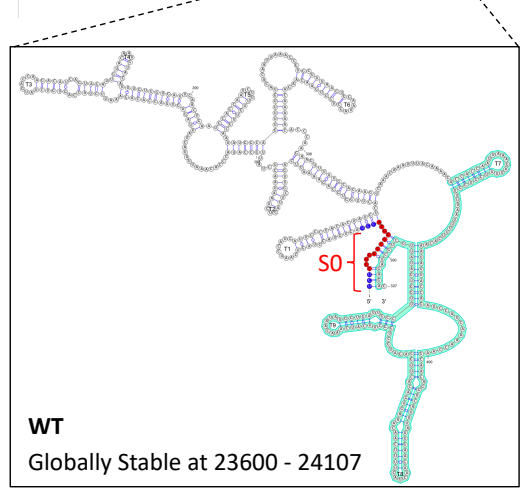

E)

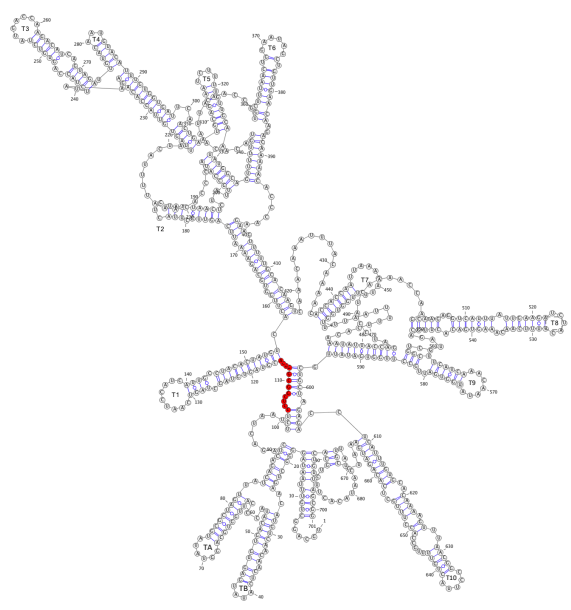

WT

Locally Stable at $23500-24200$

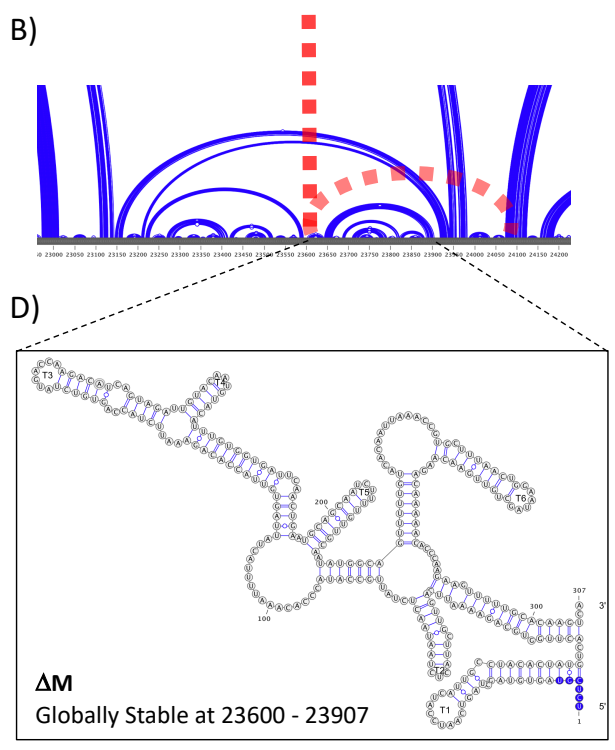

F)

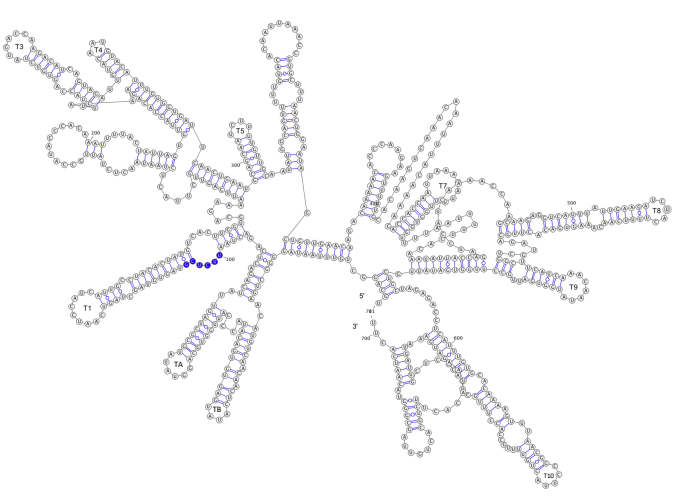

$\Delta \mathbf{M}$

Locally Stable at $23500-24200$

Figure 2. Secondary structural difference between WT and $\triangle M$ SARS-CoV-2 RNA. (A) \& (C). Predicted WT structure using global analysis (B) \& (D) Predicted $\triangle M$ structure using global analysis; Panels $C$ and $D$ depict zoomed regions around the 12-base motif CCUCGGCGGGCA. (E) Predicted WT structure using local analysis of $\sim 500$ bases encompassing the 12-base motif CCUCGGCGGGCA; (E) Predicted WT structure using the local analysis of 700 bases encompassing the 12-base motif CCUCGGCGGGCA; (F) Predicted $\triangle \mathrm{M}$ structure using the local analysis of 700 bases encompassing the original location of the deleted 12-base motif CCUCGGCGGGCA. Red color indicates the 12-base motif CCUCGGCGGGCA. Stem SO partially includes the 12-base motif and shows the pairing between regions [23603-23611] and [24097 - 24103]. Blue color indicates the nucleotides flanking the 12-base motif. Solid and broken red ribbons show the position of 12-base motif in WT and deleted 12-base motif in $\Delta \mathrm{M}$, respectively. Green ribbon indicates the stem loops that appear in the global prediction of WT but not in $\triangle \mathrm{M}$. 


\section{Discussion}

Most current studies are centered around SARS-CoV-2 proteins (e.g. Spike) and their host protein partners (e.g. ACE2). Much less attention has been given to the RNA genome of this novel virus and its potential role in viral transmission and pathogenicity. Here we show that the inserted 12-base motif may have an additional, and perhaps more important role, which is related to the SARS-CoV-2 RNA secondary structure. Using global and local RNA structure modelling, we observed that the 12-base motif forms a stable RNA secondary structure, and deletion of this motif is associated with a dramatic change in the RNA secondary structure (Figures 1 and 2). On the other hand, point mutations within this motif is predicted not to have a significant effect (Figure 1). Given the large size of SARS-CoV-2 RNA ( 30,000 bases), we acknowledge that our global analysis may not correctly predict all secondary structures, and further computational and experimental analyses are needed to validate the predicted interactions. Nonetheless, it is rather remarkable that only two structural conformations with distinct features are predicted for the WT and seven mutant RNA sequences studies here. Secondary RNA structures are known to play a critical role in viral life cycle by affecting the expression and splicing of transcripts ${ }^{24-26}$, RNA-protein interactions ${ }^{27}$, RNA editing ${ }^{28}$, and other molecular processes ${ }^{29}$. A recent study has shown that SARS-CoV-2 transcriptome is highly complex and includes numerous novel transcripts and modifications ${ }^{30}$. It is possible that the inserted 12-base motif also induces secondary structure changes in SARS-CoV-2 transcripts, which in turn enhances their translation and interaction with the host components necessary for viral replication and immune evasion. Our data serves to highlight the need for future studies to focus on the RNA at both genomic and transcriptomic levels to better understand the molecular sources of viral pandemics such as COVID-19. 


\section{References}

1 Bendavid, E. et al. COVID-19 Antibody Seroprevalence in Santa Clara County, California. medRxiv, doi:doi: https://doi.org/10.1101/2020.04.14.20062463 (2020).

2 Andersen, K. G., Rambaut, A., Lipkin, W. I., Holmes, E. C. \& Garry, R. F. The proximal origin of SARS-CoV-2. Nat Med 26, 450-452, doi:10.1038/s41591-020-0820-9 (2020).

3 Yan, R. et al. Structural basis for the recognition of SARS-CoV-2 by full-length human ACE2. Science 367, 1444-1448, doi:10.1126/science.abb2762 (2020).

4 Lan, J. et al. Structure of the SARS-CoV-2 spike receptor-binding domain bound to the ACE2 receptor. Nature, doi:10.1038/s41586-020-2180-5 (2020).

5 Shang, J. et al. Structural basis of receptor recognition by SARS-CoV-2. Nature, doi:10.1038/s41586-020-2179-y (2020).

6 Perlman, S. \& Netland, J. Coronaviruses post-SARS: update on replication and pathogenesis. Nat Rev Microbiol 7, 439-450, doi:10.1038/nrmicro2147 (2009).

7 Weiss, S. R. Forty years with coronaviruses. J Exp Med 217, doi:10.1084/jem.20200537 (2020).

8 Zhou, P. et al. A pneumonia outbreak associated with a new coronavirus of probable bat origin. Nature 579, 270-273, doi:10.1038/s41586-020-2012-7 (2020).

9 Lam, T. T. et al. Identifying SARS-CoV-2 related coronaviruses in Malayan pangolins. Nature, doi:10.1038/s41586-020-2169-0 (2020).

10 Coutard, B. et al. The spike glycoprotein of the new coronavirus 2019-nCoV contains a furin-like cleavage site absent in CoV of the same clade. Antiviral Res 176, 104742, doi:10.1016/j.antiviral.2020.104742 (2020).

11 Walls, A. C. et al. Structure, Function, and Antigenicity of the SARS-CoV-2 Spike Glycoprotein. Cell 181, 281-292 e286, doi:10.1016/j.cell.2020.02.058 (2020).

$12 \mathrm{Ou}, \mathrm{X}$. et al. Characterization of spike glycoprotein of SARS-CoV-2 on virus entry and its immune cross-reactivity with SARS-CoV. Nature communications 11, 1620, doi:10.1038/s41467-020-15562-9 (2020). 
13 Hoffmann, M. et al. SARS-CoV-2 Cell Entry Depends on ACE2 and TMPRSS2 and Is Blocked by a Clinically Proven Protease Inhibitor. Cell 181, 271-280 e278, doi:10.1016/j.cell.2020.02.052 (2020).

14 Hoffmann, M., Kleine-Weber, H. \& Pöhlmann, S. A multibasic cleavage site in the spike protein of SARS-CoV-2 is essential for infection of human lung cells. Mol Cell, in press, doi:DOI: 10.1016/j.molcel.2020.04.022 (2020).

15 Lau, S. Y. et al. Attenuated SARS-CoV-2 variants with deletions at the S1/S2 junction. Emerg Microbes Infect, 1-15, doi:10.1080/22221751.2020.1756700 (2020).

16 Chan, J. F. et al. Genomic characterization of the 2019 novel human-pathogenic coronavirus isolated from a patient with atypical pneumonia after visiting Wuhan. Emerg Microbes Infect 9, 221-236, doi:10.1080/22221751.2020.1719902 (2020).

17 Rangan, R., Zheludev, I. N. \& Das, R. RNA genome conservation and secondary structure in SARS-CoV-2 and SARS-related viruses. bioRxiv, doi:doi: https://doi.org/10.1101/2020.03.27.012906 (2020).

18 Long, S. D. \& Pekala, P. H. Regulation of GLUT4 mRNA stability by tumor necrosis factoralpha: alterations in both protein binding to the $3^{\prime}$ untranslated region and initiation of translation. Biochem Biophys Res Commun 220, 949-953, doi:10.1006/bbrc.1996.0512 (1996).

19 Lamping, E., Niimi, M. \& Cannon, R. D. Small, synthetic, GC-rich mRNA stem-loop modules 5 ' proximal to the AUG start-codon predictably tune gene expression in yeast. Microb Cell Fact 12, 74, doi:10.1186/1475-2859-12-74 (2013).

20 Courel, M. et al. GC content shapes mRNA storage and decay in human cells. Elife 8, doi:10.7554/eLife.49708 (2019).

21 Hofacker, I. L. et al. Fast folding and comparison of RNA secondary structures. Monatshefte für Chemie / Chemical Monthly 125, 167-188, doi:https://doi.org/10.1007/BF00818163 (1994). 
22 Zuker, M. \& Stiegler, P. Optimal computer folding of large RNA sequences using thermodynamics and auxiliary information. Nucleic Acids Res 9, 133-148, doi:10.1093/nar/9.1.133 (1981).

23 Darty, K., Denise, A. \& Ponty, Y. VARNA: Interactive drawing and editing of the RNA secondary structure. Bioinformatics 25, 1974-1975, doi:10.1093/bioinformatics/btp250 (2009).

24 Ilyinskii, P. O. et al. Importance of mRNA secondary structural elements for the expression of influenza virus genes. OMICS 13, 421-430, doi:10.1089/omi.2009.0036 (2009).

25 Szlachta, K. et al. Alternative DNA secondary structure formation affects RNA polymerase II promoter-proximal pausing in human. Genome Biol 19, 89, doi:10.1186/s13059-0181463-8 (2018).

26 Buratti, E. \& Baralle, F. E. Influence of RNA secondary structure on the pre-mRNA splicing process. Mol Cell Biol 24, 10505-10514, doi:10.1128/MCB.24.24.10505-10514.2004 (2004).

27 Witteveldt, J. et al. The influence of viral RNA secondary structure on interactions with innate host cell defences. Nucleic Acids Res 42, 3314-3329, doi:10.1093/nar/gkt1291 (2014).

28 Tian, N. et al. A structural determinant required for RNA editing. Nucleic Acids Res 39, 5669-5681, doi:10.1093/nar/gkr144 (2011).

29 Brigham, B. S., Kitzrow, J. P., Reyes, J. C., Musier-Forsyth, K. \& Munro, J. B. Intrinsic conformational dynamics of the HIV-1 genomic RNA 5'UTR. Proc Natl Acad Sci U S A 116, 10372-10381, doi:10.1073/pnas.1902271116 (2019).

30 Kim, D. et al. The architecture of SARS-CoV-2 transcriptome. Cell, doi:DOI: 10.1016/j.cell.2020.04.011 (2020). 


\section{Acknowledgement}

This study is supported by a Pilot Project Grant on Development of New SARS-CoV-2 Treatments from the University of Texas Health Science Center at San Antonio. 